\title{
Mid-term implant survival, functional and radiological results and mechanical complications of mega-prosthetic reconstruction around the knee with the PENTA ${ }^{\circledast}$ system
}

\author{
Harzem Özger ${ }^{1} \cdot$ Bugra Alpan $^{2}(D) \cdot$ Ahmet Salduz $^{6} \cdot$ Volkan Gurkan $^{3} \cdot$ Mustafa Sungur $^{4} \cdot$ Natig Valiyev $^{5} \cdot$ Levent Eralp $^{1}$
}

Received: 17 September 2020 / Accepted: 28 July 2021 / Published online: 21 August 2021

(c) The Author(s) 2021

\begin{abstract}
Aim Mega-prosthetic reconstruction is the most common treatment method for massive osteoarticular defects caused by tumor resection around the knee. The new implant is a highly modular rotational-hinged megaprosthesis system with a distinct pentagonal stem geometry and variable implantation options. The aim of this study is to present the mid-term implant survival characteristics, functional and radiological results and mechanical complication profile of the new megaprosthesis. Methods One hundred and one mega-prosthetic knee reconstruction procedures in 90 patients (M/F: 51/39) utilizing the new implant system were retrospectively analyzed. In 68 patients, the megaprosthesis was used for primary reconstruction following tumor resection while it was used for revision of other implants in 22. The mean age was 28.5 (7-66) years and the mean follow-up was 59.2 (24-124) months. The most common primary pathology was osteosarcoma with 63-70\% patients, the most common anatomical site of involvement was the distal femur with 56-62\% patients.

Results Henderson Type 2 failure (aseptic loosening) was seen in only 2-2.2\% patients while Type 3 (structural failure) was seen in 29-32.2\% Although the 5-year anchorage survival rate was 94.3\%, overall mechanical implant survival was $76.1 \%$ at 5 years due to a relatively high failure rate in the first-generation hinge mechanism of the implant. The 5-year hinge survival rate demonstrated a significant improvement rate from $61.7 \%$ to $87.2 \%$ between the first and second generations of the implant ( $p=0.027$ ). The mean MSTS score was 24 out of 30 (14-29). The mean cumulative ISOLS radiographic score for index megaprosthesis operations was 19.7 (12-24), which corresponded to excellent outcome.

Conclusion The new megaprosthesis system is a reliable choice for the reconstruction of tumor-related massive osteoarticular defects around the knee. Although long-term follow-up is necessary for a definitive evaluation of the implant's survival characteristics, midterm follow-up yields exceptional anchorage properties related to pentagonal stem geometry with very good functional outcomes.
\end{abstract}

Keywords Limb salvage $\cdot$ Bone neoplasms $\cdot$ Surgical oncology $\cdot$ Knee prosthesis $\cdot$ Reconstructive surgical procedure $\cdot$ Prosthesis failure $\cdot$ Prosthesis survival $\cdot$ Knee joint

\section{Introduction}

While advances in imaging modalities, oncologic treatments and surgical technique have set the ground for limb salvage surgery to become the mainstay of management in musculoskeletal tumors over the last four decades, improved implant design has been particularly instrumental in providing better quality of life to limb salvage

Bugra Alpan

balpan@gmail.com

Extended author information available on the last page of the article patients. Modular mega-prosthetic implants are the most common means of non-biological reconstruction around the knee joint following tumor resection. While these implants facilitate early weight-bearing and rapid restoration of function [1-5], the complex biomechanics of the knee, the loss of static and dynamic periarticular soft tissue stabilizers due to tumor resection and the high-demand use of the limb associated with young age contribute to mega-prosthetic failure through various non-oncological mechanisms with longer follow-up [1, 4-7]. The design and manufacturing qualities of a mega-prosthetic knee implant as well as the surgical technique directly impact the longevity of reconstruction and the quality of life. 
Taking into consideration the disadvantageous aspects of previous locally manufactured implants and national difficulties in import issues, PENTA modular extremity reconstruction system was co-developed by the senior author (HO) and TIPSAN (Izmir, Turkey) orthopedic device company. Minimizing mega-prosthetic failure requiring major revision and maximizing limb function were the design goals. The implant was named after the pentagonal cross section of its stem and was made available for the local market in 2009 with CE certification. The implant's hinge design was modified in 2011 and the 2ndgeneration hinge mechanism was used from 2012 onwards.

This study aims to evaluate the mid-term implant survival rate, functional results, radiological results and the mechanical complications of mega-prosthetic reconstruction around the knee with PENTA $®$ system.

\section{Patients and methods}

\section{Study population}

The study was conducted as a retrospective analysis based on the tumor registries of 3 different orthopedic oncology centers following approval by institutional review board. A total of 188 consecutive patients were found to have undergone megaprosthesis reconstruction, including all anatomic locations (knee, hip, shoulder) between 2009 and 2020, during which PENTA system was used exclusively for mega-prosthetic reconstruction in all unless a partial revision of a different implant was being performed. Exclusion of patients with less than 2 years of follow-up and/or operated for non-neoplastic conditions yielded 101 PENTA mega-prosthetic knee reconstruction procedures in 90 (M/F: 51/39) patients. Provided having a minimum 2-year followup, 11 additional surgeries performed in 10 out of these 90 patients due to structural PENTA failure were included in the study (Table 1). Operations were performed by 4 different surgeons; $49-54 \%$ patients were operated by the senior author (HO). While $80-89 \%$ patients had a malignant primary pathology, the most common primary pathology was osteosarcoma with $63-70 \%$ patients. The primary tumor was located in the distal femur in 56-62\% patients and proximal tibia in 34-38\%. In 68-76\% patients, PENTA was used for primary reconstruction following tumor resection while it was used for revision of other implants in $22-24 \%$. Twelve out of these 22 patients underwent revision for aseptic loosening, 5 for structural failure and 5 for chronic deep infection. PENTA was implanted in the second stage of revision in the infection cases. The 1st-generation hinge design was used in the index PENTA procedure in $28-31 \%$ patients, while the 2nd-generation hinge was used in index surgery of
$62-69 \%$ patients. The mean age was 28.5 (7-66) years at the time of index PENTA surgery and the mean follow-up was 59.2 (24-124) months. Fifty-four (60\%) patients received oncological (chemotherapy, radiotherapy or both) treatment peri-operatively with regard to the index PENTA surgery. All patients underwent routine staging and follow-up procedures pre- and postoperatively (eg. plain radiography, MRI, CT chest, PET/CT or whole-body MRI).

\section{PENTA modular extremity reconstruction system}

PENTA is a set of implants designed to reconstruct irreparable defects involving the hip, knee, shoulder and elbow joints caused by tumor resection and revision of megaprostheses. All metallic components of the implant are manufactured from Ti6Al4V except for the femoral component for proximal tibia resection prosthesis, which is made of CoCrMo. The inserts and bushings in the joint mechanism are made of ultra-high molecular weight polyethylene (UHMW-PE) [8]. The design rationale and main advantages of the implant system can be described in relation to its anchorage, modularity and articulation characteristics.

Anchorage The name of the implant is derived from the pentagonal cross-section of its stem. The stem is slightly tapered towards the tip to prevent both unnecessary bone loss and stress shielding. Although the stem design particularly favors cement-less implantation, both hydroxyapatite (HA)-coated and rough-sanded stem options are available for cement-less and cemented implantation. The pentagonal stem geometry is aimed at combining the advantages

Table 1 Demographic features of the patients

\begin{tabular}{ll}
\hline Number of patients & 90 \\
Number of surgeries & 101 \\
Mean age (years) & $28.5(7-66)$ \\
Mean follow-up (months) & $59.2(24-124)$ \\
Primary diagnosis of the patients & \\
Osteosarcoma & $63(70.0 \%)$ \\
Chondrosarcoma & $6(6.7 \%)$ \\
Ewing's Sarcoma & $7(3.3 \%)$ \\
Other malignant (PS, FS, SS, lymphoma, met Ca) & $8(8.9 \%)$ \\
Benign aggressive (GCTB, DF, ChB, ABC) & $10(11.1 \%)$ \\
Localization of primary tumor & \\
Distal femur & $56(62.2 \%)$ \\
Proximal tibia & $34(37.8 \%)$ \\
Setting of PENTA surgery & \\
Primary implant following tumor resection & $68(76 \%)$ pts \\
Revision implant & $22(24 \%)$ pts \\
\hline
\end{tabular}

$P S$ pleomorphic sarcoma, $F S$ fibrosarcoma, SS synovial sarcoma, met $C a$ metastatic carcinoma, GCTB giant cell tumor of bone, $D F$ desmoplastic fibroma, $C h B$ chondroblastoma, $A B C$ aneurysmal bone cyst, pts patients 
of square and hexagonal cross-sectional stems, which are increased primary rotational stability and decreased risk of bone damage during cement-less implantation, respectively. Furthermore, HA-coating improves primary stability against pull-out forces by increasing friction at the bone-implant interface and ensures secondary stability through osseo-integration. A wide array of stem options are present, in terms of stem length (120-150-200 mm) stem diameter (12-22 mm) and stem curvature (straight or anatomical), to guarantee the best anchorage in all intraoperative scenarios. All stems have collars to prevent subsidence. The stem collars are also HA-coated to promote extracortical bone bridging and thus better secondary stability.

Modularity PENTA megaprosthesis system has a highly modular design to allow fine-tuning of the reconstruction in terms of limb length and rotational alignment. The limb length can be adjusted at $1-\mathrm{cm}$ intervals with modular extension components. On the other hand, connections between the modular parts are secured with 3 different features; toothed-connection between all parts, conical press-fit connection between the parts and axial trans-fixation bolt, which spans the length of all components on each side of the joint. Distal femoral bodies for distal femoral resection and femoral components for proximal tibial resection have $5^{\circ}$ of valgus. On the other hand, proximal tibial bodies for proximal tibial resection and the tibial baseplates for distal femoral resection are built without laterality. Size options are available for these modular parts.

Articulation PENTA knee megaprosthesis has a rotational hinge mechanism, which connects the femoral and tibial components through a yoke assembly (tibial rotation piece). The hinge formed by the distal femur and the yoke allows a $0^{\circ}-130^{\circ}$ range of motion in the sagittal plane. While hyperextension was solely blocked by a thick bumper insert in the first-generation hinge mechanism, the design was modified and a second-generation hinge was used from 2012 onwards. The 2 nd-generation hinge, which is still currently in use, limits maximum flexion and extension by corresponding stepped rotation blocks on the axle head and inside the axle socket of the femoral component. The sharp edge of the intercondylar notch, which came into contact with the bumper insert during hyperextension, was also rounded off in the 2 nd-generation hinge. The yoke can rotate $15^{\circ}$ both internally and externally through its articulation with the proximal tibia. Rotation occurs around the cylindrical yoke post, which resides unconstrained in the proximal tibia. However, rotation is actually limited by 2 features. The yoke has a convex undersurface with 2 projections while the concave upper surface of the tibial insert, which is fixed to the proximal tibia, has 2 grooves corresponding to the projections under the yoke. The interface geometry of these two non-spherical components ensures a smooth stop at the endpoints of rotation, where the under-projections are limited by the grooves (Fig. 1).

\section{Postoperative rehabilitation and follow-up}

Isometric quadriceps exercises were started as soon as adequate analgesia was achieved. Postoperatively, prophylactic intravenous antibiotics were used in all cases until the removal of all drains. The drains were removed at an average of 4 days. Primary cases were allowed full weight-bearing immediately after surgery with two crutches. However, weight-bearing was protracted up to 6 months in revision cases with graft impaction. Following proximal tibial resections, knee immobilizers were used for 6 weeks to allow for healing of the extensor mechanism. Knee flexion was allowed after 6 weeks with the goal to obtain $15^{\circ}$ of flexion for every two weeks. Patients were followed up every three months for the first 2 years, every 6 months for the next three years and annually after 5 years.

\section{Evaluation methods}

Endo-prosthetic reconstruction failures were classified according to failure modes described by Henderson et al. [9]. Any soft tissue complication requiring invasive procedures ranging from hematoma aspiration to flap reconstruction was accepted as Type 1 failure. Prosthetic stems with radiographically and clinically shown macro-motion in the absence of septic clinical, radiological and laboratory findings were accepted as having Type 2 failure. Clinical pathological movement and/or any radiographic finding of loss of structural integrity were accepted as Type 3 failure. Patients with chronic pain in anchorage sites regardless of radiographic appearance or with stem loosening in the presence of laboratory findings, with peri-prosthetic abscess formation or chronic fistula and with culture-positive periprosthetic effusions were accepted as having Type 4 failure. Patients with clinically and/or radiologically detected and biopsy proven lesions in the vicinity of megaprosthesis were accepted as having Type 5 failure. All 5 types of failure were screened for and both mechanical and non-mechanical failures were reported for a comprehensive overview of the study population. However, the outcome analysis focused on mechanical (Type 2 and 3) failures, in conformity with the aim of the study. Mechanical failures were established with clinical and radiological findings. Survival analysis of the implant was done with regard to overall mechanical survival, anchorage survival and hinge mechanism survival. Anchorage failure was designated as any breakage or aseptic loosening of the anchorage components (including stem, tibial baseplate of the distal femoral replacement or femoral component of proximal tibial replacement). Hinge failure was designated as any breakage of the hinge mechanism 
Fig. 1 Design features of the PENTA ${ }^{\circledR}$ are shown. A and $\mathbf{B}$ Demonstrate the pentagonal, hydroxyapatite-coated stem. C and $\mathbf{D}$ Demonstrate the rotational ability in both directions and the curbed-stop mechanism with the hinge distracted. $\mathbf{E}$ and F Demonstrate the extension and flexion blockage by the stepped rotation blocks on the axle head and inside the axle socket of the femoral component. G Demonstrates the proximal tibia body articulating with the femoral component

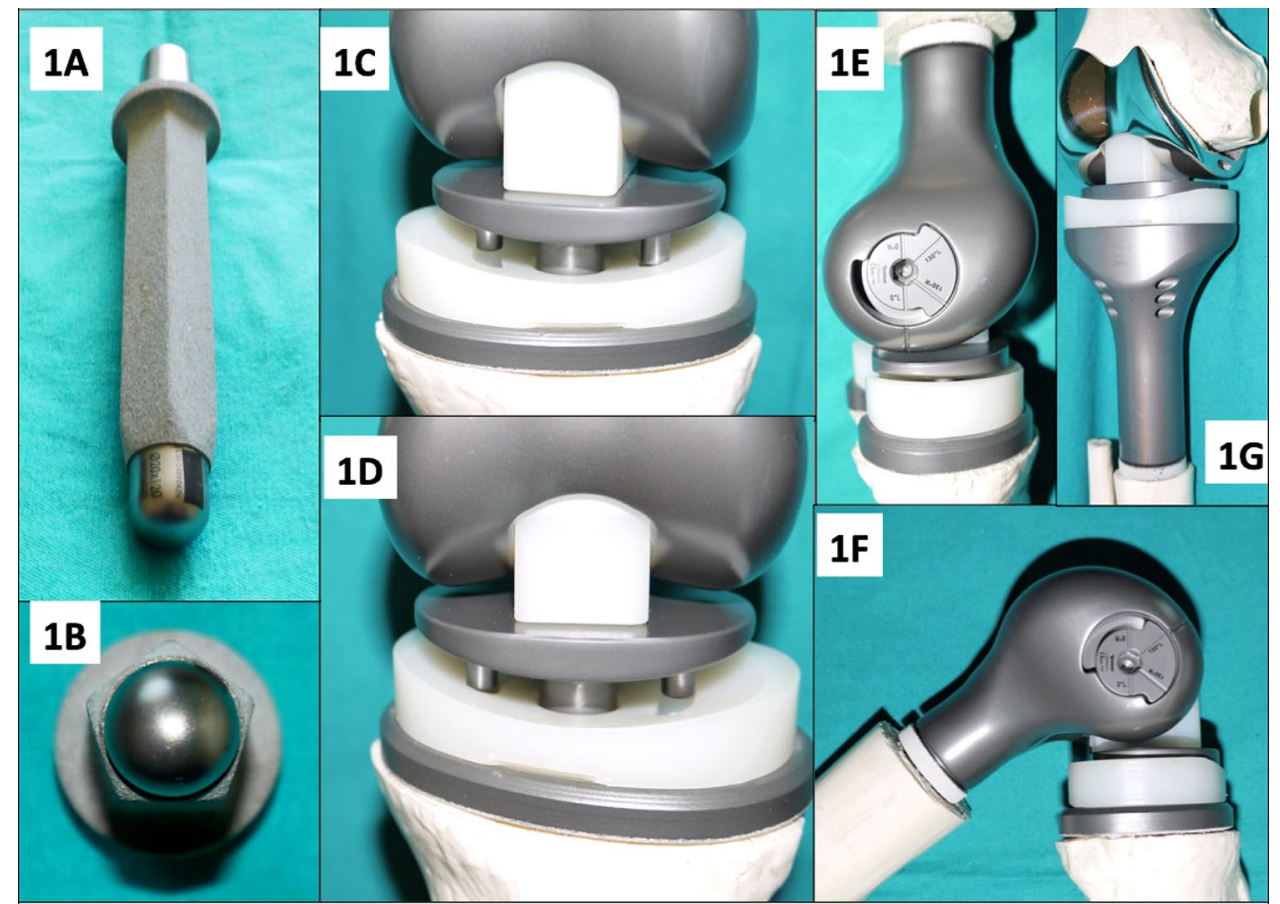

components (including bumper insert, axle, axle bushing, tibial rotation piece, tibial insert). Any revision surgery, which had already been performed or was being planned at the time of data collection, was considered as the endpoint of survival for both anchorage and hinge components. For patients with multiple modes of mechanical failure, the overall mechanical survival was determined according to whichever failure developed first.

Radiological outcomes were assessed according to the ISOLS radiographic scoring system for implants (bone remodeling, interface, anchorage, implant body, implant articulation, extracortical bone bridging) [10]. Each radiographic parameter was scored as 1 (poor), 2 (fair), 3 (good) or 4 (excellent). The radiographic scores were used both individually to objectively identify specific failure types (Type 2; Type 3-hinge failure, anchorage breakage, periprosthetic fracture) and also collectively to give an overall score for the implant. Peri-prosthetic fractures were also assessed according to the Unified Classification System (UCS), which provides a better understanding of the relationship of the implant to the fracture.

Functional outcomes were evaluated using Musculoskeletal Tumor Society (MSTS) Scoring System [11].

Statistical analysis was done using SPSS 20 (IBM). Descriptive statistics of the patients, survival of the implants, and the association of various clinical variables and failure of the implants were analyzed using Kaplan-Meier survivorship curve, log-rank test and Cox proportional hazards regression model.

\section{Results}

\section{Functional outcome}

The mean MSTS was 23.8 (14-29) out of 30 including all patients who underwent index PENTA procedure. MSTS scores were significantly better after primary PENTA procedures $(24.6 \pm 2.9)$ compared to revision PENTA procedures $(21.4 \pm 3.7)(p=0.001)$. MSTS scores were also significantly better after distal femoral reconstructions $(24.6 \pm 3.3)$ compared to proximal tibia reconstructions $(22.5 \pm 3.2)$ $(p=0.003)$. The mean MSTS score for patients who underwent primary PENTA procedure with the 2nd-generation implant was $24.7 \pm 3.2$. Seventy-eight patients with good to excellent radiographic interface scores had a mean MSTS score of $24.2 \pm 3.2$, which was significantly higher when compared to the mean score of $21.7 \pm 3.8$ for 12 patients with poor to fair interface scores according to paired samples test $(p=0.000)$. MSTS scores did not correlate with any other demographic parameter, mechanical mode of failure, generation of implant (1st vs. 2nd) or overall ISOLS radiographic score. Although clinical symptoms of knee discomfort were resolved after revision of failed hinge mechanisms, MSTS scores did not demonstrate a significant change.

\section{Radiological outcome}

The mean overall ISOLS radiographic implant score was $19.7 \pm 2.9$ out of 24 for all patients following the index PENTA operation. The mean overall radiographic score 
was significantly higher $(20.3 \pm 2.8)$ in patients who were implanted with the 2nd-generation PENTA prosthesis in the index operation compared to the mean score $(18.5 \pm 2.5)$ of patients implanted with the 1st generation $(p=0.005)$. Patients who underwent primary PENTA procedure with the 2nd-generation implant had the highest mean overall radiographic score of $20.8 \pm 2.5$ out of 24 .

The mean scores for each radiographic subcategory were as follows: bone remodeling $(3.2 \pm 1.0)$. interface $(3.4 \pm 0.6)$. anchorage (3.8 \pm 0.7$)$. implant body (4.0). implant articulation $(3.1 \pm 1.3)$ and extracortical bone bridging $(2.2 \pm 1.1)$ out of 4 points. Thus, 5 out of 6 radiographic parameters demonstrated good to excellent outcomes while extracortical bone bridging was fair to good. The mean implant articulation score was significantly higher ( 3.5 out of 4 ) with the 2nd-generation implant compared to the mean of 1.8 out of 4 with the 1 st-generation implant $(p=0.000)$ (Fig. 2).

\section{Megaprosthesis complications}

Soft tissue failure, Type 1: This was observed in 20-22.2\% patients. Most of soft tissue failures occurred as in the form of hematoma/seroma formation, wound dehiscence or superficial necrosis. These problems were treated with early aggressive debridement and vacuum-assisted closure techniques. In two patients, hematoma and patella subluxation occurred and were treated by debridement, medial plication and lateral release. In 3 patients, treatment required free flaps. No patient in this group required implant revision attributable to these complications and none of these complications were regarded as having a causal relationship with the use of PENTA implant.

Aseptic loosening, Type 2: Aseptic loosening was observed around the femoral stems in $2-2.2 \%$ patients who had primarily been treated for osteosarcoma and had undergone revision surgery with PENTA in the setting of aseptic loosening of another implant. Both cases had required extensive bone graft impaction on the femoral side during revision surgery due to loss of bone stock and enlargement of the medullary cavity. Bone remodeling, interface and extracortical bone bridging scores were poor for both patients. Resorption of impacted graft material, thus biological insufficiency, seemed to be the cause of aseptic loosening for these cases.

Structural failure Type 3: Structural failure was observed in $29-32.2 \%$ patients. A total of 19 hinge failures, 7 periprosthetic fractures and 5 anchorage breakages were observed in these 29 patients.

Patients with a poor radiographic "implant articulation" score were uniformly symptomatic and described either mediolateral, posterior or rotationary laxity, clunky sounds and either pain or discomfort in their knee joints. Hinge failure was observed in 13 out of $28-46,4 \%$ procedures with the 1st-generation PENTA implant and in 8 out of $73(11,1 \%)$ procedures with the 2 nd-generation implant. While hinge failures were significantly more common in proximal tibia
Fig. 2 17-year-old female osteosarcoma patient underwent right distal femoral replacement with PENTA® following neoadjuvant chemotherapy. A Orthoroentgenograms at 5 years postoperatively. B Plain radiographs at the same follow-up demonstrate the bone remodeling around the stem
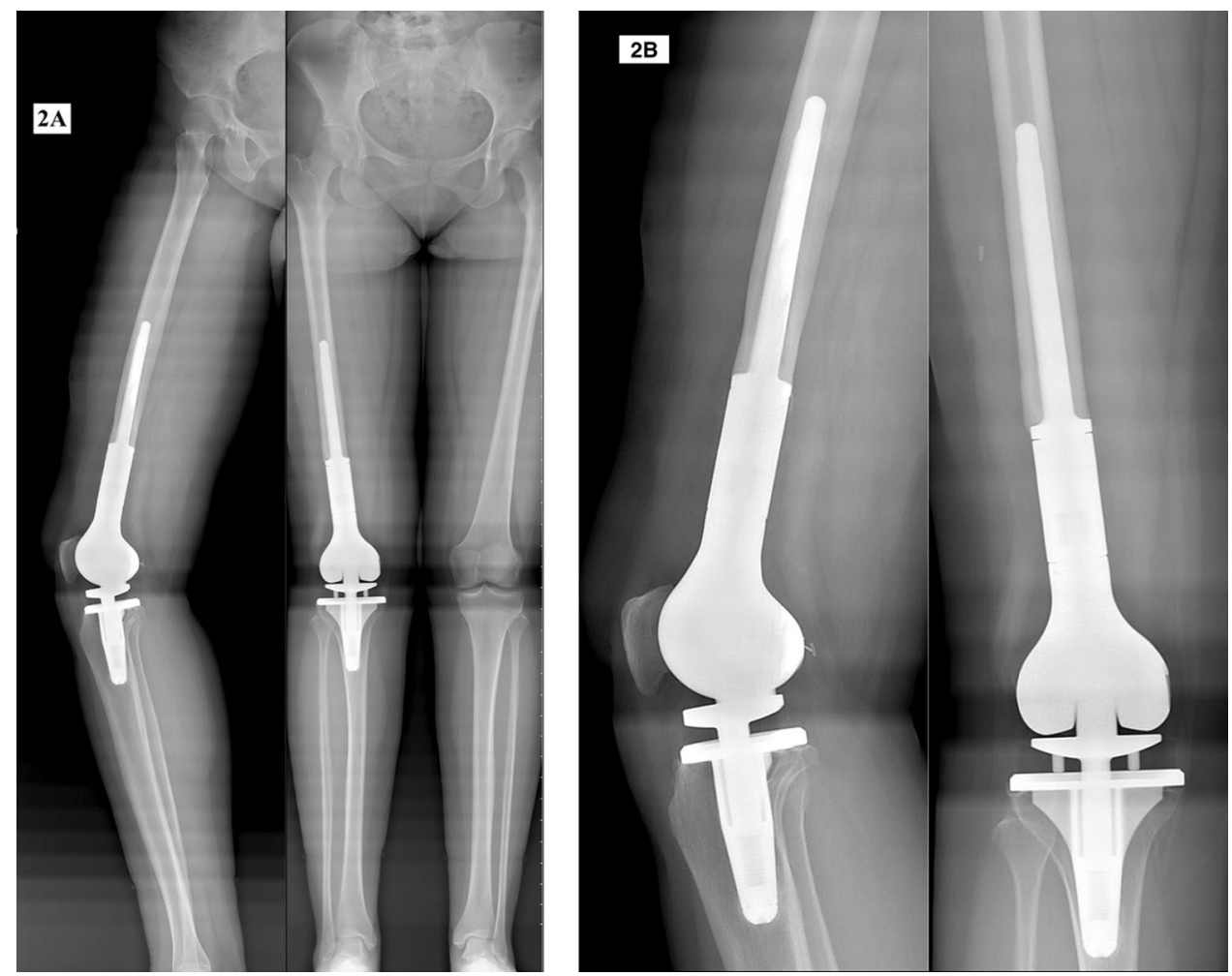
cases $(p=0.020)$, no other significant etiologic relationship could be demonstrated with any other demographic data.

Although periprosthetic fracture was encountered in 7 patients, the stem was retained in all cases. The ISOLS radiographic score for "bone remodeling" was recorded as poor "by definition" in 5 and as good in 2 out of these 7 patients. On the other hand, according to the UCS classification, 5 were type B1 (fracture around the stem, with the stem stable) and 2 were Type $C$ (fracture in the same bone but away from the implant). Both Type $\mathrm{C}$ patients and one patient with type $\mathrm{B} 1$ required osteosynthesis while the rest were managed conservatively.

Four broken femoral stems were revised and a tibial baseplate with extension stem awaited revision, while this study was prepared. While the "anchorage" scores were uniformly poor "by definition" in these patients, their mean "bone remodeling" and "extracortical bone bridging" scores (1.8 and 1.0 out of 4.0 , respectively) were also significantly lower than the mean scores for all patients ( 3.2 and 2.3 out of 4.0 , respectively) ( $p=0.001, p=0.009$ ). These low radiographic scores were caused by lack of bony contact around the stem under the stem collar. Retrospective analysis of early postoperative radiographs and surgical notes revealed that femoral stems were prematurely fixed inside the medullary canal during insertion with part of the stem shaft and collar left unsupported in 4 patients while the impacted bone grafts were resorbed leaving the tibial baseplate unsupported in the remaining patient. The stem diameter was $11 \mathrm{~mm}$ in all broken femoral stems. The breakage occurred at the junction of baseplate and extension stem in the tibial case. The interface scores were good in all indicating that the supported stem segments were well fixed. Although 6 out of 7 mechanical stem failures were seen in distal femur and only 1 in proximal tibia, the correlation was not significant.

Infection, Type 4: Peri-prosthetic deep infection was observed in 5-5.6\% patients. One patient was treated with debridement, revision of hinge mechanism including polyethylene parts, antibiotic-loaded cement beads and soft tissue reconstruction with latissimus dorsi free flap. Other four patients were treated with intravenous antibiotics, debridement and vacuum-assisted closure. Two of these patients developed chronic fistula and intractable infection. They were then offered trans-femoral amputation but refused amputation. These patients were still ambulatory with the implants clinically and radiologically stable despite ongoing infection at the last follow-up.

Local recurrence, Type 5: Local recurrence was observed in $6-6,7 \%$ patients ( 5 malignant, 1 benign). One patient with fibrosarcoma underwent trans-femoral amputation. Five patients were treated with wide re-excision retaining the implants. Four of these 6 patients died with lung metastases.

The failure modes are summarized in Table 2.

\section{Implant survival analysis}

The overall mechanical 5-year survival rate (including Type $2 \& 3$ failures) was $81.3 \%$ (47 patients) and $92.3 \%$ (15 patients) for the 2nd-generation PENTA as primary or revision implant, respectively. On the other hand, 5-year overall mechanical survival rate was $76.1 \%$ for all index PENTA operations in 90 patients (Fig. 3A). The 5-year hinge survival rate was $80.4 \%$ for all 101 PENTA procedures. However, the 5-year hinge survival rate was $89.8 \%$ for procedures with the 2nd-generation hinge mechanism and this was significantly better than $63.9 \%$ 5-year hinge survival rate for procedures with the 1st-generation hinge mechanism $(p=0.041)$ (Fig. 3B). The 5-year and 10-year anchorage survival rates were, respectively, $94.3 \%$ and $79.8 \%$ including all index PENTA operations (Fig. 3C). Anchorage survival rates did not demonstrate significant difference between primary and revision surgeries, 1 st and 2 nd generations of the implant and whether or not hinge failure occurred.

\section{Oncological outcome}

Distant metastases were observed in $15-16.7 \%$ patients and $10-11.1 \%$ patients died of disease during follow-up.

\section{Discussion}

As life expectancy and rate of limb salvage in patients with primary malignant bone tumors increase, the durability of the reconstruction becomes more important. Durability of the endo-prostheses is closely related to their design features. While hinge mechanism revisions are more common due to the wear of mobile parts, failure and subsequent revision of anchorage components cause greater morbidity associated with tumor prostheses.

The censored data of excluded patients (30 out of 120 PENTA knee reconstruction patients) might be considered as a limitation of this study. While 4 out of these 30 were operated for non-neoplastic conditions and compromised the homogeneity of the study population, the remaining 26

Table 2 Failure modes and frequencies

\begin{tabular}{ll}
\hline Failure mode & $\begin{array}{l}\text { Number } \\
\text { of patients } \\
(\%)\end{array}$ \\
\hline Type 1 (soft tissue failure) & $20(22.2)$ \\
Type 2 (aseptic loosening) & $2(2.2)$ \\
Type 3 (structural failure) & $29(32.2)$ \\
Type 4 (infection) & $5(5.6)$ \\
Type 5 (local recurrence) & $6(6.7)$ \\
\hline
\end{tabular}



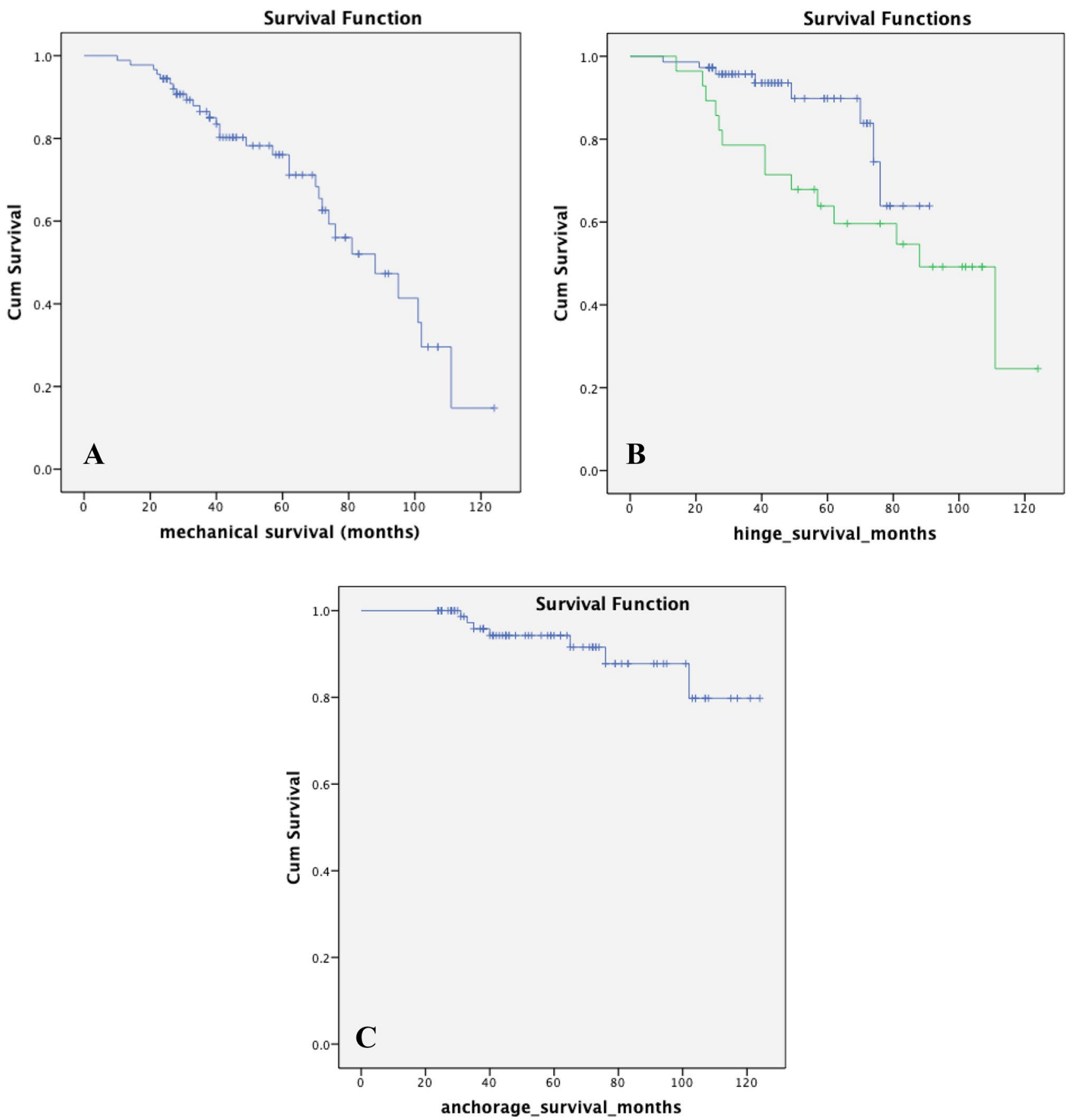

Fig. 3 Mid-term Kaplan-Meier survival analyses of the PENTA®. A 5-year overall mechanical survival graph. B 5-year hinge survival graph (The green and blue lines represent 1st and 2nd generations, respectively). C 5-year anchorage survival graph

were excluded either because they died of disease or were lost to follow-up due to logistic and/or socioeconomic reasons, before 2 years. Since the majority of patients undergoing mega-prosthetic knee reconstruction are those with malignant musculoskeletal tumors, limited follow-up due to death is a natural occurrence in such a cohort. While the insufficient data of patients, which were left out, might decrease the statistical power of the study, we believe that 
the outcome analyses are not skewed by our exclusion criteria. Revision PENTA procedures as well as primary procedures were included in the study since all revision procedures were performed on patients, who had also initially undergone limb salvage for oncological reasons and endoprosthetic interventions are afflicted by common problems, such as previous oncological treatment, defective and weakened bone stock, weak soft tissue coverage, and extensive muscle loss in both groups.

A brief review of global mega-prostheses used over the last decades yields several major designs and implant systems (Table 3). In Kotz Modular Femur-Tibia Reconstruction System (KMFTR), diaphyseal fixation was obtained with screws going through the 2 lateral flanges [12]. This design usually failed through progressive wear of the polyethylene and loosening, and stem breakage at the site of the nearest screw hole [13-15]. Howmedica Modular Reconstruction System (HMRS) had an improved design over KMFTR and had a single flange to reduce stress shielding $[13,16,17]$. Ruggieri et al. evaluated both of these fixed hinge prostheses [18] and showed $4.8 \%$ breakage in prostheses requiring revision (10,5\% in KMFTR, 3,5\% in HMRS). HMRS prostheses had a significantly lower rate of breakage compared to KMFTR. Aseptic loosening rates were not found to be significantly different (4.9\% in HMRS, 9.6\% in KMFTR). Global Modular Reconstruction System (GMRS) is an improvement on HMRS with its rotating hinge. Rotating hinge decreased mechanical stresses and complications at the bone-implant interface. Aseptic loosening rates (5\%), although decreased with the introduction of rotating hinges remain a common cause implant failure $[4,18]$.

Dynamic compression fixation, the philosophy behind Compress Pre-Stress Implant (Biomet Inc, Warsaw, IN), was a novel idea to reduce osteolysis in the proximal tibia and distal femur. These stems provided stem stabilization without the need for long stems, decreased stress shielding and osteolysis and increased osteointegration. Most common complications of this implant were aseptic loosening (9.7\%) and fracture of the underlying bone between the anchor plug and the spindle $[19,20]$.

While cement-less stems offer the crucial advantage of osteointegration and are therefore expected to yield better survival outcomes with regard to aseptic loosening, some authors advocate the use of cemented prostheses mainly due to better stress-shielding properties. The survival outcomes of Stanmore prostheses with cemented stems have been studied extensively over long follow-up periods in large study populations. Unwin et al. reported aseptic loosening as the

Table 3 Outcomes and survival in major distal femoral and proximal tibial megaprosthetic reconstruction series

\begin{tabular}{|c|c|c|c|c|c|c|c|c|c|}
\hline Literature & Site, brand & Follow-up (m) & $N$ & Type $1(n, \%)$ & Type $2(n, \%)$ & Type $3(n, \%)$ & Type $4(n, \%)$ & Type $5(n, \%)$ & $\begin{array}{l}\text { 5-year } \\
\text { implant } \\
\text { survival } \\
(\%)\end{array}$ \\
\hline \multirow[t]{2}{*}{ Pala et al. [4] } & DF (GMRS) & $48(24-96)$ & 187 & $13(6.9)$ & $10(5.3)$ & 0 & $16(8.6)$ & $11(5.9)$ & \\
\hline & PT (GMRS) & $48(24-96)$ & 60 & $8(13.3)$ & $4(6.7)$ & 0 & $7(11.7)$ & $3(5.0)$ & \\
\hline \multirow{2}{*}{$\begin{array}{l}\text { Gosheger } \\
\text { et al. [6] }\end{array}$} & DF (Mutars) & $45(3-140)$ & 103 & - & $15(14.6)$ & $3(2.8)$ & $12(11.7)$ & - & 66 \\
\hline & PT (Mutars) & & 42 & - & $3(7.1)$ & $1(2.4)$ & $7(16.7)$ & - & 62 \\
\hline \multirow{2}{*}{$\begin{array}{l}\text { Ruggieri et al. } \\
{[18]}\end{array}$} & HMRS & $132(24-300)$ & 543 & & $26(4.9)$ & $19(3.5)$ & $45(8.4)$ & & $80(10 \mathrm{yr})$ \\
\hline & KMFTR & & 126 & & $12(9.6)$ & & & & \\
\hline $\begin{array}{l}\text { Heisel et al. } \\
{[24]}\end{array}$ & Mutars & $46(24-84)$ & 50 & - & $11(22)$ & $5(10)$ & $6(12)$ & - & - \\
\hline \multirow[t]{2}{*}{$\begin{array}{l}\text { Capanna et al. } \\
\text { [29] }\end{array}$} & $\begin{array}{l}\text { DF (megasys- } \\
\text { tem) }\end{array}$ & $67(24-149)$ & 87 & $4(4.6)$ & $3(3.4)$ & $18(20.7)$ & $12(13.8)$ & $4(4.6)$ & 70 \\
\hline & $\begin{array}{l}\text { PT (megasys- } \\
\text { tem) }\end{array}$ & $67(24-149)$ & 32 & $3(9.4)$ & 0 & 0 & $3(9.4)$ & $2(2.3)$ & 84 \\
\hline $\begin{array}{l}\text { Myers et al. } \\
{[30]^{*}}\end{array}$ & $\begin{array}{l}\text { DF (Stan- } \\
\text { more) }\end{array}$ & $144(60-360)$ & 173 & & $41(24)$ & - & $32^{* *}(9.6)$ & - & $83^{* *}$ \\
\hline $\begin{array}{l}\text { Myers et al. } \\
{[31]^{*}}\end{array}$ & $\begin{array}{l}\text { PT (Stan- } \\
\text { more) }\end{array}$ & $176(60-348)$ & 99 & & $3(3)$ & & $37^{\&}(19.5)$ & & $68^{\mathbb{I}}$ \\
\hline \multirow[t]{2}{*}{ Current study } & DF (PENTA) & $60(24-121)$ & 56 & $13(23.2)$ & $1(1.8)$ & $15(26.8)$ & $3(5.4)$ & $6(10.7)$ & 91 \\
\hline & PT (PENTA) & $56(24-124)$ & 34 & $7(20.6)$ & $1(2.9)$ & $14(41.2)$ & $2(5.9)$ & 0 & 100 \\
\hline
\end{tabular}

$D F$ distal femur, $P T$ Proximal tibia

* Only rotating hinge group is depicted

** Out of 335 patients

II Out of 194 patients 
most common cause of failure with 10-year stem survival rates of $67.4 \%$ and $54.8 \%$, respectively, in distal femur and proximal tibia in a series with 1001 patients. Coathhup et al. on the other hand, reported significantly improved 10-year survival of $88.9 \%$ against aseptic loosening with the use of HA-coated collars on cemented stems in Stanmore distal femur prostheses [21-23].

MUTARS (Implancast, Germany) was introduced to the market in 1995. It can be inserted cemented or press-fit and has a hexagonal cross section. This hexagonal design contributed to good rotational stability and low rates of aseptic loosening (8\%) according to a 250 patient series by Gosheger et al. [6]. The deep infection rate was found to be $12 \%$ and stem breakage rate was $1.6 \%$ in the same study. Another study on 100 patients by Heisel et al. showed an aseptic loosening rate of $22 \%$ and deep infection rate of $12 \%$ [24].

The aseptic loosening rate of $2.2 \%$ in our study series was remarkably lower compared to other established prosthesis designs. loosening rate was $2.2 \%$. This outcome can be attributed to HA-coated pentagonal, slightly tapered and collared stems, which provide exceptional primary and secondary stability. Radiological criteria support this successful outcome with regard to anchorage, bone remodeling and bone-implant interface scores.

Although no clear support in the literature exists regarding cemented and cement-less fixation, some authors suggested that cement-less fixation might be advantageous due to bone integration and lower aseptic loosening rates. To increase osteointegration between implant and the surrounding bone, cement-less press-fit stems have been constructed in different shapes (fluted, fenestrated) and different textures (grit-blasted, porous-coated, beaded, hydroxy-apatite coated). In their 232 patient study, Pala et al. compared cemented and cement-less fixation and observed cementless fixation group to have higher overall survival and survival to infection whereas survival to aseptic loosening was not significantly different than the cemented group [25]. Cemented fixation may be appropriate in patient with bone metastases, extensive osteolytic defects, bad prognosis and elderly patients. In young patients with primary bone tumors, cement-less fixation is preferable [14, 25]. In this study un-cemented PENTA stems resulted in excellent midterm survival.

To achieve the best results, the prosthesis must respect the biomechanics. Early tumor prosthesis designs with fixed hinges failed due to torsional forces on the anchorage sites, which resulted in aseptic loosening. Newer designs with rotating hinges tried to emulate the physiological rotation of the knee joint through the arc of motion. These rotating hinge designs also had their share of problems. This sudden stop in some cases resulted in loosening around the junction of stem and body, which further led to implant breakage and peri-prosthetic fracture. We have encountered such cases in designs with a single canal in polyethylene for rotation. Another possible design for rotating hinges uses elevation of the plateau during rotation as a screw-home mechanism; however, if prosthesis continues to rotate, dislocation can occur. PENTA has two canals in the polyethylene insert for rotation. This allows for load distribution at the ends of rotation. The PE insert of PENTA has a concave surface to improve contact surface with femoral condyles and increase stability with a smooth stop.

Implant failure includes both stem revision and revision of joint mechanism. First-generation PENTA designs had issues with early wear of the bumper in hyperextension. The wear of the bumper was followed by metal abrasion and cracking or wear of the axle bushing, which eventually led to global instability. The sharp edge of the femoral notch, which damaged the bumper insert was beveled and hyperextension loads were also distributed through stepped rotation blocks on the axle head and inside the axle socket of the femoral component with a design change. High body mass index and excessive hyperextension loading during ambulation due to extensor muscle loss may have contributed to the wear of the polyethylene bumper in these patients.

Peri-prosthetic deep infection is a major mode of failure in patients with knee mega-prostheses. Coating implant with silver was introduced as a sophisticated and promising strategy against this complication in 2000s. Successful prevention and treatment of peri-megaprosthetic joint infections in oncological patients undergoing surgery for different anatomic locations have been reported in both original studies and meta-analyses [26, 27]. While the infection rate for patients undergoing primary megaprosthetic reconstruction with silver-coated implants ranged from 9 to $10 \%$, a significantly reduced re-infection rate of $13.7-29.2 \%$ was reported when silver-coated implants were used instead of uncoated implants in revision surgery [26, 27]. On the other hand, another review of the literature by authors, who have long advocated and have substantial experience with the use of silver-coated implants, emphasizes that long-term definitive evidence is needed to show effectiveness of silver and it is only expected to prevent biofilm formation [28]. Deep infection rate was remarkably low in our study population when compared with infection rates from the literature (Table 3 ). Although this excellent outcome might be attributed to the PENTA implant, periprosthetic infection is obviously multifactorial and cannot be evaluated independent from variables like surgical technique and management of soft tissues. 


\section{Conclusion}

PENTA megaprosthesis system is a reliable choice for the reconstruction of tumor-related massive osteoarticular defects around the knee. The results demonstrate that the design issue of the 1st-generation hinge was resolved in the 2nd generation. Although long-term follow-up is necessary for a definitive evaluation of the implant's survival characteristics and performance, short to mid-term follow-up yields exceptional anchorage properties with very good functional outcomes.

Acknowledgements The senior author (HO) holds a patent license for the implant described and used in the study. He is also a non-paid consultant for the company manufacturing the implant. The other authors of this study or any member of their immediate family, has no funding or commercial associations (eg, consultancies, stock ownership, equity interest, patent/licensing arrangements, etc.) that might pose a conflict of interest in connection with the submitted article. This study was assessed by the Institutional Review Board of Istanbul University Istanbul Medical Faculty Dept. of Orthopaedics and Traumatology and approved on compliance with the rules of ethics and scientific guidelines.Authors would like to thank Ms. Hicran Dumlu for her contributions in data collection and co-ordination of the study.

\section{Declarations}

Conflict of interest The authors declare that they have no conflict of interest.

Open Access This article is licensed under a Creative Commons Attribution 4.0 International License, which permits use, sharing, adaptation, distribution and reproduction in any medium or format, as long as you give appropriate credit to the original author(s) and the source, provide a link to the Creative Commons licence, and indicate if changes were made. The images or other third party material in this article are included in the article's Creative Commons licence, unless indicated otherwise in a credit line to the material. If material is not included in the article's Creative Commons licence and your intended use is not permitted by statutory regulation or exceeds the permitted use, you will need to obtain permission directly from the copyright holder. To view a copy of this licence, visit http://creativecommons.org/licenses/by/4.0/.

\section{References}

1. Ahlmann ER, Menendez LR, Kermani C, Gotha H (2006) Survivorship and clinical outcome of modular endoprosthetic reconstruction for neoplastic disease of the lower limb. J Bone Joint Surg Br 88:790-795. https://doi.org/10.1302/0301-620X.88B6. 17519

2. Mirra JM (1989) Bone tumors: clinical, radiologic, and pathologic correlations. Lea \& Febiger, Philadelphia

3. Pala E, Trovarelli G, Angelini A, Maraldi M, Berizzi A, Ruggieri P (2017) Megaprosthesis of the knee in tumor and revision surgery. Acta Biomed 88(2S):129-138. https://doi.org/10.23750/ abm.v88i2-S.6523

4. Pala E, Trovarelli G, Calabrò T, Angelini A, Abati CN, Ruggieri $P$ (2015) Survival of modern knee tumor megaprostheses: failures, functional results, and a comparative statistical analysis.
Clin Orthop Relat Res 473:891-899. https://doi.org/10.1007/ s11999-014-3699-2

5. Ruggieri P, Bosco G, Pala E, Errani C, Mercuri M (2010) Local recurrence, survival and function after total femur resection and megaprosthetic reconstruction for bone sarcomas. Clin Orthop Relat Res 468:2860-2866. https://doi.org/10.1007/ s11999-010-1476-4

6. Gosheger G, Gebert C, Ahrens H, Streitbuerger A, Winkelmann W, Hardes J (2006) Endoprosthetic reconstruction in 250 patients with sarcoma. Clin Orthop Relat Res 450:164-171. https://doi.org/10.1097/01.blo.0000223978.36831.39

7. Zeegen EN, Aponte-Tinao LA, Hornicek FJ, Gebhardt MC, Mankin HJ (2004) Survivorship analysis of 141 modular metallic endoprostheses at early followup. Clin Orthop Relat Res 420:239-250

8. PENTA MERS - Surgical Technique Implants \& Instruments. Available at: http://www.tipsan.com.tr/pdf/teknikler/TIPSAN OP_TEK_pentamers.pdf

9. Henderson ER, Groundland JS, Pala E, Dennis JA, Wooten R, Cheong D et al (2011) Failure mode classification for tumor endoprostheses: retrospective review of five institutions and a literature review. J Bone Joint Surg Am 93:418-429. https://doi. org/10.2106/JBJS.J.00834

10. Langlais F, Tomeno B (2012) Limb Salvage: Major Reconstructions in Oncologic and Nontumoral Conditions. 5th International Symposium, St. Malo ISOLS-GETO. New York, NY: Springer-Verlag

11. Enneking WF, Dunham W (1993) A system for the functional evaluation of reconstructive procedures after surgical treatment of tumors of the musculoskeletal system. Clin Orthop and Relat Res 286:241-246

12. Mittermayer F, Windhager R, Dominkus M, Krepler P, Schwameis E, Sluga M et al (2002) Revision of the Kotz type of tumour endoprosthesis for the lower limb. J Bone Joint Surg Br 84:401-406. https://doi.org/10.1302/0301-620X.84B3.08404 01

13. Campanacci M, Capanna R, Ruggieri P, Ritschl P, De Cristofaro R, Ferraro A, De Maio M (1990) Cementless modular prostheses. Results and complications. Chir Organi Mov 75(1 Suppl):179-183

14. Griffin AM, Parsons JA, Davis AM, Bell RS, Wunder JS (2005) Uncemented tumor endoprostheses at the knee: root causes of failure. Clin Orthop Relat Res 438:71-79. https://doi.org/10.1097/ 01.blo.0000180050.27961.8a

15. Kotz R (2005) The development of a modular tumor endoprosthesis (KMFTR-HMRS-GMRS). Evolution, results and perspectives. Arch Ortop Reum. 116:9-11

16. Ilyas I, Kurar A, Moreau PG, Younge DA (2001) Modular megaprosthesis for distal femoral tumors. Int Orthop 25:375-377. https://doi.org/10.1007/s002640100290

17 Lan F, Wunder JS, Griffin AM, Davis AM, Bell RS, White LM, Ichise M, Cole W (2000) Periprosthetic bone remodelling around a prosthesis for distal femoral tumours. Measurement by dualenergy X-ray absorptiometry (DEXA). J Bone Joint Surg Br. 82:120-125. https://doi.org/10.1302/0301-620x.82b1.9563

18. Ruggieri P, Mavrogenis AF, Pala E, Abdel-Mota'al M, Mercuri M (2012) Long term results of fixed-hinge megaprostheses in limb salvage for malignancy. Knee 19:543-549. https://doi.org/10. 1016/j.knee.2011.08.003

19. Bini SA, Johnston JO, Martin DL (2000) Compliant prestress fixation in tumor prostheses: interface retrieval data. Orthopedics 23:707-712

20. Healey JH, Morris CD, Athanasian EA, Boland PJ (2013) Compress $®$ knee arthroplasty has $80 \% 10$-year survivorship and novel forms of bone failure. Clin Orthop Relat Res 471:774-783. https:// doi.org/10.1007/s11999-012-2635-6 
21. Coathup MJ, Batta V, Pollock RC, Aston WJ, Cannon SR, Skinner JA et al (2013) Long-term survival of cemented distal femoral endoprostheses with a hydroxyapatite-coated collar: a histological study and a radiographic follow-up. J Bone Joint Surg Am 95:1569-1575. https://doi.org/10.2106/JBJS.L.00362

22. Unwin PS, Cobb JP, Walker PS (1993) Distal femoral arthroplasty using custom-made prostheses: the first 218 cases. J Arthroplasty 8:259-268. https://doi.org/10.1016/s0883-5403(06)80087-2

23. Unwin PS, Cannon SR, Grimer RJ, Kemp HB, Sneath RS, Walker PS (1996) Aseptic loosening in cemented custom-made prosthetic replacements for bone tumours of the lower limb. J Bone Joint Surg British 78(1):5-13

24. Heisel C, Kinkel S, Bernd L, Ewerbeck V (2006) Megaprostheses for the treatment of malignant bone tumours of the lower limbs. Int Orthop 30:452-457. https://doi.org/10.1007/s00264-006-0207-7

25. Pala E, Mavrogenis AF, Angelini A, Henderson ER, Douglas Letson G, Ruggieri P (2013) Cemented versus cementless endoprostheses for lower limb salvage surgery. J BUON 18:496-503

26. Schmolders J, Koob S, Schepers P, Pennekamp PH, Gravius S, Wirtz DC et al (2017) Lower limb reconstruction in tumor patients using modular silver-coated megaprostheses with regard to perimegaprosthetic joint infection: a case series, including 100 patients and review of the literature. Arch Orthop Trauma Surg 137(2):149-153. https://doi.org/10.1007/s00402-016-2584-8

27 Fiore M, Sambri A, Zucchini R, Giannini C, Donati DM, De Paolis M (2020) Silver-coated megaprosthesis in prevention and treatmentof peri-prosthetic infections: a systematic review and meta-analysis about efficacy and toxicity in primary and revision surgery. Eur J Orthop Surg Traumatol. https://doi.org/10.1007/ s00590-020-02779-Z

28 Schmidt-Braekling T, Streitbuerger A, Gosheger G, Boettner F, Nottrott M, Ahrens H et al (2017) J Silver-coated megaprostheses: review of the literature. Eur J Orthop Surg Traumatol. https://doi. org/10.1007/s00590-017-1933-9

29. Capanna R, Scoccianti G, Frenos F, Vilardi A, Beltrami G, Campanacci DA (2015) What was the survival of megaprostheses in lower limb reconstructions after tumor resections? Clin Orthop Relat Res 473:820-830. https://doi.org/10.1007/ s11999-014-3736-1

30. Myers GJ, Abudu AT, Carter SR, Tillman RM, Grimer RJ (2007) Endoprosthetic replacement of the distal femur for bone tumours: long-term results. J Bone Joint Surg Br 89:521-526. https://doi. org/10.1302/0301-620X.89B4.18631

31. Myers GJ, Abudu AT, Carter SR, Tillman RM, Grimer RJ (2007) The long-term results of endoprosthetic replacement of the proximal tibia for bone tumours. J Bone Joint Surg Br 89:1632-1637. https://doi.org/10.1302/0301-620X.89B12.19481

Publisher's Note Springer Nature remains neutral with regard to jurisdictional claims in published maps and institutional affiliations.

\title{
Authors and Affiliations
}

\author{
Harzem Özger ${ }^{1} \cdot$ Bugra Alpan $^{2} \mathbb{E} \cdot$ Ahmet Salduz $^{6} \cdot$ Volkan Gurkan $^{3} \cdot$ Mustafa Sungur $^{4} \cdot$ Natig Valiyev $^{5} \cdot$ Levent Eralp $^{1}$ \\ Harzem Özger \\ harzemo@yahoo.mail.com \\ Ahmet Salduz \\ ahmetsalduz@gmail.com \\ Volkan Gurkan \\ volgur@hotmail.com \\ Mustafa Sungur \\ drmsungur@gmail.com \\ Natig Valiyev \\ natigvaliyev@gmail.com \\ Levent Eralp \\ drleventeralp@gmail.com \\ 1 Istanbul Faculty of Medicine, Department of Orthopaedics \\ and Traumatology (Retired), Istanbul University, \\ 2 School of Medicine, Department of Orthopaedics \\ and Traumatology, Acibadem Mehmet Ali Aydinlar \\ University, Kayisdagi, Cad. 34752 Atasehir, Istanbul, Turkey \\ 3 Medical Faculty, Department of Orthopaedics \\ and Traumatology, Bezm-i Alem Vakif University, Fatih, \\ Istanbul, Turkey \\ 4 Department of Orthopaedics and Traumatology, \\ AcibademAtakent Hospital, Turgut Ozal Bulvari No:16, \\ 34303 Kucukcekmece, Istanbul, Turkey \\ 5 Department of Orthopaedics and Traumatology, Acibadem \\ Maslak Hospital, Buyukdere Caddesi No:40, 34457 Sariyer, \\ Istanbul, Turkey \\ 6 Istanbul Faculty of Medicine, Department of Orthopaedics \\ and Traumatology , Istanbul University, Capa 34093 Fatih, \\ Istanbul, Turkey
} Capa 34093 Fatih, Istanbul, Turkey 